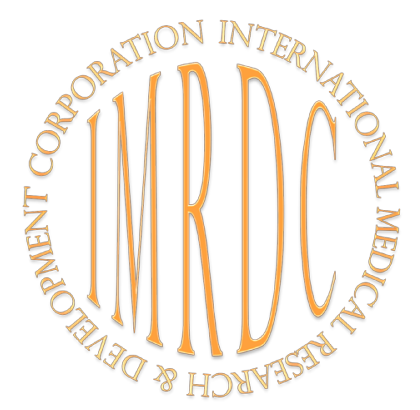

\title{
Functional Bowel Disorders and Obesity in Children: State of the Problem
}

\author{
Anna Pogodina, PhD, ScD*; Anastasia Romanitsa, PGS; \\ Lyubov Rychkova, $\mathrm{PhD}, \mathrm{ScD}$, Corresponding Member of the RAS \\ Scientific Centre for Family Health and Human Reproduction Problems \\ Irkutsk, the Russian Federation
}

\begin{abstract}
Obesity and functional bowel disorders (FBDs) are often observed in children and have common risk factors. The present review aimed to summarize the published data on the association between obesity and FBDs in children and a discussion of possible pathophysiological mechanisms that may be involved. Published data indicates that obesity and FBDs could be associated conditions. There is substantial evidence that obesity in children is associated with constipation. However, it should be noted that there were few studies in this direction, and those studies were heterogeneous in both the composition of participants and studied diagnostic criteria, and in the majority of cases, they were not adjusted for potential confounders. The association between obesity and FBDs can be explored through diet, peculiarities of eating behavior, and psychological factors. The most promising direction in the study could be the study of the gut microbiota, the changes in which can contribute to the development of immune dysfunctions of the bowel, chronic low-grade inflammation, increased colonic permeability, motility disturbances, and visceral hypersensitivity. The studies in this area can provide important data for developing a strategy of treatment and prevention of both groups of diseases. (International Journal of Biomedicine. 2020;10(4):316-323.)
\end{abstract}

Key Words: irritable bowel syndrome • functional constipation • adolescents

\section{Abbreviations}

BW, body weight; GM, gut microbiota; FBD, functional bowel disorders; FGIDs, functional gastrointestinal disorders; IBS, irritable bowel syndrome; FC, functional constipation.

$\mathrm{O}$ besity in children is one of the most serious problems in public healthcare of the 21 st century. From 1980 to 2013, the number of adults who were overweight or obese increased by $27.5 \%$ and children - by $47.1 \% .^{(1)}$ The results of a multicenter study in Russia showed that around $20 \%$ of children were overweight and 5\% suffered from obesity.(2) It is a concerning fact that children with obesity continue to suffer from it in adult life. If the existing tendencies remain, around $20 \%$ of infants with obesity will turn into children with obesity, $40 \%$ of children with obesity will turn into adolescents with obesity, and $80 \%$ of these adolescents will inevitably become adults with obesity. ${ }^{(3)}$ Comorbid conditions

*Corresponding author: Anna Pogodina, PhD, ScD. Scientific Centre for Family Health and Human Reproduction Problems, Irkutsk, the Russian Federation.E-mail:pogodina_av@inbox.ru with obesity include a wide spectrum of chronic diseases that involve nearly all organs and can exert a significant long-term negative effect on health and life expectancy. Thus, in 2015, there were 4 million obesity-related deaths and 120 million disability-adjusted life years in the world. ${ }^{(3)}$

Even though the main efforts of researchers are focused on the study of the associations between obesity and such potentially life-threatening conditions as diabetes mellitus, cardiovascular diseases, and nonalcoholic fatty liver disease, recently, data has appeared that shows an association between obesity and functional gastrointestinal disorders (FGIDs) in adult and pediatric populations. FGIDs are a heterogeneous group of recurrent abdominal symptoms, the origin of which does not have a structural or biochemical explanation. ${ }^{(4)}$ They are widespread in the pediatric practice, i.e. $50 \%$ of visits to pediatric gastroenterologists and $2-4 \%$ of general pediatric visits. $^{(5)}$ 
Rome IV criteria approved in 2016 divided pediatric FGIDs into two groups: group $G$ that included disorders observed in children from birth to 4 years old (not included in the review) and group $\mathrm{H}$ that included disorders in children older than 4 years and adolescents. The last group contains three categories: functional disorders associated with nausea and vomiting, functional disorders associated with abdominal pain, and functional defecatory disorders ${ }^{(6)}$ FBDs that are characterized by a change in the rate of defecation and stool consistency, as well as IBS and FC, are the most common reasons for seeking treatment.

The majority of studies dedicated to the association of obesity and FBDs were conducted in adult cohorts. Pediatric studies in this area are sparse and provide controversial results. Along with that, obesity and FBDs seem to have more in common than a high incidence rate in the population. In the pathogenesis of both conditions, there are factors of diet and lifestyle, psychological impairments, and altered GM. Thus, an understanding of the true associations between obesity and FBDs in pediatric patients is important for providing key information for the development of pathogenetically grounded strategies of treatment, prevention, and early diagnostics for both groups of diseases.

Thus, the present review aimed to summarize the published data on the association between obesity and FBDs in children and a discussion of possible pathophysiological mechanisms that may be involved.

\section{Definitions}

It should be noted that the definition of FBDs in children and adolescents changed after the approval of the Rome IV criteria.

IBS is diagnosed in children with abdominal pains that persist for not less than 4 days per month. At the same time, they are associated with defecation, change in the rate and consistency of stool, and in combination, these symptoms cannot be explained by other medical conditions. The specified complaints should persist for not less than two preceding months. Based on the prevailing stool shape, IBS is divided phenotypically into IBS with constipation (IBS-C), IBS with diarrhea (IBS-D), a mixed subtype of IBS and IBS without a subtype. ${ }^{(7)}$ However, it should be noted that in $24 \%$ of children, the subtype of IBS can change within 12 months. ${ }^{(8)}$ This could be associated with the fact that the majority of studies on IBS in pediatrics do not consider separate subtypes of this disease and look into IBS as one group.

FC in children older than 4 years old is diagnosed in cases with two or more symptoms, provided they are observed not less than one time per week for minimum of one month: two or fewer defecations per week; at least one episode of fecal inconsistency per week; a history of retentive posturing or excessive volitional stool retention; a history of painful or hard bowel movements; the presence of a large fecal mass in the rectum; a history of large-diameter stools that may obstruct the toilet. At the same time, the specified symptoms cannot be explained by other medical causes. ${ }^{(9)}$

It should be highlighted that according to the Rome IV criteria, abdominal pain does not exclude FC. However, in children with FC, unlike in children with IBS, constipation can be the main symptom. Pains disappear after FC resolution and are considered to be secondary symptoms. ${ }^{(9)}$ Thus, the separation of FC and IBS-C requires thorough detailing of complaints, which is not always possible in pediatric patients. The described facts and constant evolution of diagnostic criteria of FGIDs, which has been ongoing since 1990, significantly hamper their unification for systematic reviews and meta-analyses.

Obesity and FBDs in children: Is there an association between them?

Studies that have focused on the association between FBDs and obesity can be divided into two categories: studies among patients of specialized clinics and centers, and studies performed among samplings of the general population.

The association between FBDs and obesity in children and adolescents has been primarily studied among gastrointestinal patients. Two studies performed at gastrointestinal centers in the USA $(n=375)$ and Israel $(n=274)$, with 10 years between them, that included population control groups showed that there was a significant association between these diseases despite different variants of Rome criteria used for to verify the diagnosis..$^{(10,11)}$ The study performed by Pawłowska et al. ${ }^{(12)}$ showed that IBS in pediatric patients was associated not only with excessive BW but also with the share of fat mass in the body that was assessed using bioimpedancemetry.

The rate of IBS among children with obesity was studied once, and the results showed the rates to be significantly higher than in the control group $(10.67 \%$ vs $2.60 \% ; P=0.024)$. ${ }^{(13)}$

The authors managed to find only a single, but welldesigned, study that focused on the incidence rate of FGIDs, including IBS and FC, in the sampling of healthy children aged 4-18 years old $(n=450)$. The study was conducted in a general pediatric clinic without a bias towards either obesity or FGIDs, which would inevitably occur at a specialized center. That study was unique because it accounted for such possible confounders as the age of the participants, their ethnicity, and the area where the participants were included in the study. The obtained results confirmed that children with obesity were more prone to the development of FGIDs, and in particular IBS, than children with healthy BW..$^{(14)}$

An association between obesity and constipation has been studied more actively but the obtained results are more controversial.

In 2004, Fishman et al. ${ }^{(15)}$ were the first to report that obesity and FC are associated conditions. In further years, three studies performed in the USA showed that there was a higher incidence rate of obesity among children with constipation than among healthy children in the control group of the general population. ${ }^{(10,16,17)}$ Two of these studies showed that the association between obesity and constipation was more significant among boys. ${ }^{(16,17)}$

The association between weight status and FC was reported in three studies, which were conducted among children with obesity. ${ }^{(13,18,19)}$ Two studies, which used Rome III criteria to verify the diagnosis, presented comparable data on the rate of constipation in children with obesity $(18.44 \%$ and $21 \%$, respectively). ${ }^{(13,18)}$ In the third study, the authors did not use diagnostic validated criteria, and the rate of constipation in 
adolescents with obesity was $38.4 \%$.(19\%) It should be noted that only one of the above-mentioned studies had a control group $(23 \%$ vs $13 \%))^{(14)}$

We revealed only one study conducted among the general population, which found that children with overweight or obesity suffered from constipation more frequently than children with healthy BW.

On the other hand, several studies did not confirm the association between obesity and constipation. Among the studies that were performed in clinics, there was one conducted by Çağan Appak et al. ${ }^{(20)}$ that showed that BMI z-scores of children with FC were comparable with the control group. Another "case-control" study showed that obesity and being overweight were more common in children with FC, than in children that had other diseases $(18 \%$ vs $12 \%$ and $33 \%$ vs $23 \%$, respectively), but these differences were not statistically significant. $^{(21)}$

Three populational studies, two of which were conducted among adolescents in Brazil and one in Columbia (2820 participants aged 8-18 years old), ${ }^{(22-24)}$ also did not reveal an association between obesity and FC, though, in one of them, girls with constipation tended to have a higher BMI than girls that did not suffer from constipation. ${ }^{(24)}$ Kiefte-de Jong et al. ${ }^{(25)}$ published the results of the observation for the population cohort by year of birth $(n=2420)$. They did not reveal a significant association between excessive weight and constipation in 4-year-old children. ${ }^{(25)}$

Finally, a high-profile study that included 14,626 Taiwanese adolescents showed that there was a reverse association between rare defecations $(<3$ times per week) and being overweight or obese. ${ }^{(26)}$ Although rare defecations do not completely characterize constipation, the association between FC and a deficit of fat mass presented by Pawłowska corresponded to these results. ${ }^{(12)}$ The available data indicate that weight loss may modestly improve both upper and lower abdominal GI symptoms, thus suggesting a potential causal link. ${ }^{(27)}$

Few studies characterize the peculiarities of clinical manifestations of FBDs in children with obesity. Teitelbaum et al. ${ }^{(10)}$ showed that among all the subtypes of IBS, only IBS-D was associated with obesity in the sampling of gastroenterological patients aged 2-20 years old. This agrees with the results of the study by Gurova et al., ${ }^{(28)}$ which showed that patients aged 12-15 years old with obesity more often had unformed stool and a higher rate of defecations (up to 12-13 times per week) than adolescents who were merely overweight.

Bonilla et al. ${ }^{(29)}$ monitored the dynamics of 351 children with FGIDs associated with pain (including IBS) and revealed that obesity significantly increased the possibility of persisting abdominal pains. In children with obesity, abdominal pains occurred more often and were more intense, and they led to absence from school and routine life disturbances. When it comes to constipation, Misra et al. ${ }^{(17)}$ showed that the rate of defecations in general and the rate of painful defecations in children with FC did not depend on body weight. The same study showed that constipation in patients with obesity was more difficult to treat. There is evidence that FC in children with obesity was associated with episodes of fecal incontinence. ${ }^{(15,22)}$ However, other studies did not confirm this association. ${ }^{(16,17)}$

To sum up, the published data present quite uniform conclusions on the association between obesity and IBS but controversial conclusions on the association between obesity and FC. However, even though the association between obesity and IBS was confirmed by all the conducted studies, their conclusions have some limitations. First, these studies did not include large samplings of the population and most of them did not have sufficient power. Second, they used different diagnostic criteria for FGIDs. Third, they did not consider possible confounders, like diet and peculiarities of eating behavior, that could be more significant for IBS than obesity itself. Regarding childhood obesity and FC, the association between these diseases was confirmed in the majority of studies conducted in specialized clinics, but several studies in the general population provided neutral and even directly contrasting results. This may be because of a selection bias due to the fact that patients with more severe clinical manifestations of both FBDs and obesity seek medical care, and the associations between FBDs and obesity in clinical groups may be different than in the general population. Moreover, in some studies, there were no control groups and the rate of $\mathrm{FC}$ was compared with some average populational values. Along with that, the rate of FC could be significantly different not only between different regions but also between different regions of one country. ${ }^{(23)} \mathrm{A}$ systematic review by Koppen et al. ${ }^{(30)}$ in 2016 that focused on the studies between functional defecation disorders and excessive weight in children did not provide consistent results because of heterogeneous designs, low quality of the reviewed studies, and controversial results that were obtained by the authors.

All the above-mentioned facts indicate that despite its significance, the problem of the association between obesity and FBDs remains unresolved.

Obesity and FBDs in children: Possible mechanisms of association

Some associated factors could be considered as causes of obesity in children and adolescents. For one, the qualitative and quantitative content of a diet plays an important role in the development of obesity and symptoms of FBDs. Therefore, the peculiarities of a diet that contribute to the development of both conditions can be treated as connecting links between them..$^{(31)}$ Potentially, a tendency towards the consumption of products with a low content of fiber (which stimulates the osmolarity of stool), high content of saturated fats (which stimulate the motility), and high content of fermentable carbohydrates can contribute to the development of symptoms of FBDs in these patients. $^{(32,33)}$ In a major cohort in France, Schnabel et al. ${ }^{(34)}$ studied the association between the consumption of ultraprocessed food that was characterized by a high content of fatty acids, sugar, and sodium, and a low content of fiber and FGIDs. The results showed that an increase in the consumption of ultra-processed food was associated with a significant risk of developing IBS and was also associated with a higher IBM of the participants.

The role of excessive consumption of fermentable 
carbohydrates, such as fructose, lactose, fructans, galactans, etc., in the development of the symptoms of FBDs has been actively studied in recent years. These short-chain carbohydrates contained in a wide range of food are poorly absorbed in the intestine, and their fermentation by the GM leads to the formation of carbon dioxide, hydrogen, and/ or methane. ${ }^{(35)}$ Small osmotically active molecules that are formed during the fermentation attract liquid to the small intestine and can contribute to the development of osmotic diarrhea, symptoms of bloating, pain, and discomfort.

Excessive consumption of poorly absorbable sugars, in particular, corn syrup with fructose, which are widely consumed in the Western diet, can partially explain the association between obesity and FBDs shown in some studies.

Ozaki et al. ${ }^{(36)}$ revealed malabsorption of fructose in $67.7 \%$ of children aged $4-14$ years old with functional abdominal pain, including in $90 \%$ of children with IBS included in the study. Children with malabsorption of fructose consumed more carbohydrates and high-energy foods and had higher values of BMI Z-scores. There are few studies dedicated to the selective impact of fermentable carbohydrates in forming the association between diet and the symptoms of FBDs in patients with obesity. However, the results of double-blind, randomized, controlled studies showed the effectiveness of a diet with a low content of fermentable carbohydrates in reducing gastrointestinal symptoms in a majority of children with IBS. This can indirectly indicate the role of these carbohydrates in the development of FBDs. ${ }^{(37,38)}$ Still, because of the limited scope of such a diet, studies on its long-term influence on the growth and development of children are required before it could be widely recommended in clinical practice.

An excessive amount of fats in the diet can be also associated with symptoms of FBDs in patients with obesity. ${ }^{(39)}$ Out of all nutrient ingredients, lipids are the strongest modulators of the gastrocolic reflex. ${ }^{(40)}$ It is known that lipids in food weaken the motility of the intestine, slowing down the transit of gas via the intestine and leading to abdominal bloating; on the other hand, they enhance the motility of the colon, contributing to the development of diarrhea. ${ }^{(39)}$ In a study by Levy et al.. ${ }^{(41)}$ the content of fats in the diet of adult patients with overweight and obesity had an independent positive association with an increase in the rate of stool and diarrhea in the multivariate model.

The content of fiber in a diet is associated with constipation, and the studies we reviewed provided controversial results. Çağan Appak et al. ${ }^{(20)}$ did not reveal a significant difference in the daily consumption of fiber between healthy children and patients with FC. At the same time, Macêdo et al. ${ }^{(24)}$ demonstrated that the low content of fiber in a diet was associated with constipation in female adolescents $(\mathrm{OR}=3.42$; 95\% CI: 1.08-12.06). Also, BMI in girls with constipation was higher than in girls without constipation $(P=0.001)$, which does not exclude the fact that consumption of fiber is one of the modifiers of the association between BMI and FC.

Systematic reviews of pediatric studies did not provide evidence that would allow the specialists to recommend the consumption of fiber that exceeds the daily norm for the weight and age of a child for the treatment of FC. ${ }^{(42)}$ Nevertheless, the above-mentioned data could be a useful recommendation for patients with obesity and FC to consume a sufficient amount of dietary fiber, ${ }^{(43)}$ which can be important in the prevention and treatment of both diseases.

One of the possible explanations of the association between FBDs and obesity can be pathological patterns of eating. It has been shown that impairments of eating behavior, such as compulsive and episodic overeating and different variants of limiting behavior, were common problems among children with obesity. ${ }^{(44)}$ The results of the meta-analysis of 36 studies showed that more than a quarter of children and adolescents with overweight and obesity suffered from compulsive uncontrolled overeating, ${ }^{(45)}$ which can contribute to the development of gastrointestinal symptoms. A great volume of food leads to quick gastric distention and further osmotic bolus to the intestine, which increases the capacity of physiologic adaptation. ${ }^{(46)}$ A study by Levy et al. ${ }^{(41)}$ showed that compulsive overeating had a significant association with abdominal pain and bloating in the multivariate models.

Obesity was associated not only with behavioral eating disorders but also with such psycho-emotional impairments as anxiety and depression. ${ }^{(47,48)} \mathrm{A}$ similar spectrum of psycho-emotional impairments observed in children with FBDs $^{(49-51)}$ can indicate that obesity and FGIDs had similar psychophysiological mechanisms. A longitudinal survey by Koloski et al. ${ }^{(52)}$ showed that elevated levels of anxiety in participants that did not initially have FGIDs were significant predictors of the development of FGIDs within 12 years of the observation. In this study, the predictors of the development of IBS were elevated levels of anxiety and depression. The mechanisms that link obesity, FBDs, and psycho-emotional impairments require further studies, but it is suggested that the hypothalamic-pituitary-adrenal axis is one of the key links in the pathogenesis of obesity and that this axis also takes part in the pathogenesis of FBDs. ${ }^{(53)}$

Iovino et al. ${ }^{(54)}$ used a rectal barostat to reveal that emotional instability modulated visceral perception and could contribute to the formation of visceral hypersensitivity and abdominal pain in children with obesity. It was also shown that among patients with morbid obesity, those who suffered from IBS had significantly higher levels of fatigue, anxiety, and depression as well as a lower quality of life. ${ }^{(55)}$

One of the connective links between obesity and disturbances in the rate and character of stool could be peculiarities of gut transit. A shorter time of colonic transit in relatively healthy people with obesity can be one of the explanations of the association between obesity and diarrhea in the study that included the adult population. ${ }^{(56)}$

Bouchoucha et al. ${ }^{(57)}$ conducted a study that included 354 patients with constipation. The study not only obtained similar results but also showed a reverse association between the time of rectosigmoid transit and age. The authors suggested that children should have the maximal expression of the transit delay in the rectosigmoid region. Physiological age-related alterations in colonic transit can be associated with the neuropeptide profile of the gut, change in the content and volume of interstitial cells of Cajal, ${ }^{(58)}$ and peculiarities of the microbiological landscape. The fact that the mechanisms that form the association between FBDs and obesity that involve 
gut transit differ in different periods of life, could be one of the explanations for the controversial results obtained during the studies on the association between obesity and FC that included children and adolescents. The age-related range (usually 4-18 years old) can lead to a shift in the distribution and affect the study results. This suggestion is proven by the fact that the results of the studies that focused on adolescents were comparable with the ones that involved adults. ${ }^{(29)}$ The study of cohorts by the year of birth that included 26-yearold New Zealanders $(\mathrm{n}=980)$ revealed a significant inverse association between BMI and diarrhea, which was comparable to the results of the majority of studies that included adults. ${ }^{(59)}$

In addition to the above-described facts, obesity and FBDs have another potential common pathophysiological factor that attracts researchers - GM. On the one hand, changes in the content of GM are associated with the onset and persistence of FBDs. On the other hand, they were described as an important determinant of energetic metabolism and obesity. ${ }^{(60)}$

The GM are responsible for the fermentation of undigested proteins and carbohydrates, utilization of hydrogen, and transformation of bile acids, gases, and secondary bile acids. Metabolic functions of the GM can contribute to disturbances of the intestine's immune function, chronic lowgrade inflammation, increased colonic permeability, motility disturbances, and visceral hypersensitivity. ${ }^{(7)}$

Some studies showed that patients with obesity had an increase in the ratio of Firmicutes/Bacteroidetes, which was associated with an enhanced capacity to derive energy from food due to a more active breakdown of carbohydrate complexes. ${ }^{(61,62)}$ Even though the data on the content of microbiota in patients with IBS is controversial, these patients have a similar type of dysbiosis. ${ }^{(63)}$ Saulnier et al ${ }^{(64)}$ showed that the content of microbiota correlated with the severity and rate of abdominal pain in children with IBS. Indirectly, the role of GM in the pathogenesis of FBDs can indicate the effectiveness of treating these diseases with probiotics. A meta-analysis of three randomized placebo-controlled studies revealed a significantly higher rate of response to the treatment (resolution of weakening of pains) in children with IBS that received Lactobacillus rhamnosus GG containing probiotics, in comparison with a placebo. ${ }^{(65)}$ Clinical improvement was also observed in the treatment of children with IBS with probiotics that contained VSL\#3 and a combination of three bifidobacteria. ${ }^{(66,67)}$ The mechanisms of the positive influence of probiotics on the symptoms of IBS are to be investigated. It is also unclear how the presence of obesity affects the clinical profile of IBS in probiotic treatment.

The association between GM and FC is often studied by its influence on gut transit. Even though the pathophysiological mechanisms of this influence are not established, they could be microbial modulation of the expression of genes involved in motor apparatus responses, $\mathrm{pH}$-dependent stimulation of the motility from the products of fermentation, the osmotic effect of metabolites of the microbiota and intestinal distention due to an increase in the production of intraluminal gases (carbon dioxide, hydrogen, and methane) that provoke reflexive smooth muscle contractions. ${ }^{(68)}$ The differences in the GM content between children with FC and the control group were revealed in several studies. The study by Zhu et al. ${ }^{(69)}$ showed that the differences also remained in cases when both groups of comparison consisted of children with obesity. In this study, patients with FC (the average age was 11.8 years old) had a significantly lower level of Bacteroidetes, in particular Prevotella, and the level of some types of Firmicutes, including Lactobacillus, were significantly higher than in children without constipation, while the levels of bifidobacteria were comparable. Other researchers described an increase in the level of bifidobacteria in children with FC. ${ }^{(68,70)}$ The fact that in pediatric practice probiotics usually contain lactobacilli and bifidobacteria, the levels of which are quite high in the microbiome of children with FC, can explain why Wojtyniak's meta-analysis of seven randomized controlled studies that included 515 people did not reveal any benefits from the application of probiotics for the treatment of FC in children. ${ }^{(71)}$

\section{Conclusion}

Published data indicates that obesity and FBDs are associated conditions. The presence of significant associations between obesity and IBS in children was confirmed by all the studies dedicated to this issue. There is significant evidence that obesity in the pediatric cohort is associated with constipation. However, it should be noted that there were few studies in this direction. They were heterogeneous in the composition of participants and applied diagnostic criteria. In rare cases, the data was adjusted for possible confounders and, thus, the association between obesity and FBDs was not obvious.

The above-mentioned facts provide grounds for highquality studies with unified definitions, diagnostic criteria, precise criteria for study inclusion and exclusion, and a wide range of confounder factors, including diet, psychological, and social-demographic factors. The establishment of a true association between obesity and FBDs would contribute to a better understanding of the complicated biology of both conditions and could optimize approaches to therapy.

The study of GM as a central link that explores the association between eating habits, energy extraction, and weight gain, on one hand, and intestinal motility and visceral sensitivity, on the other, could be quite promising in the study of the association between obesity and FBDs. The studies on GM could help reveal the species of bacteria that contribute to the development of certain types of FBDs, which could provide grounds for the proposed target probiotic treatment.

\section{Competing Interests} interests.

The authors declare that they have no competing

\section{References}

1. Ng M, Fleming T, Robinson M, Thomson B, Graetz N, Margono $\mathrm{C}$, et al. Global, regional, and national prevalence of overweight and obesity in children and adults during 19802013: a systematic analysis for the Global Burden of Disease Study 2013. Lancet. 2014 Aug 30;384(9945):766-81. doi: 
10.1016/S0140-6736(14)60460-8.

2. Tutelyan VA, Baturin AK, Kon IY, Martinchik AN, Uglickih AK, Korosteleva MM, et al. [Prevalence of overweight and obesity in child population of Russia: multicenter study]. Pediatria. Journal named after G.N. Speransky. 2014;93(5):2831. doi:10.24110/0031-403X-2014-93-5-28-31. [Article in Russian].

3. GBD 2015 Obesity Collaborators; Afshin A, Forouzanfar MH, Reitsma MB, Sur P, Estep K et al. Health effects of overweight and obesity in 195 countries over 25 years. N Engl J Med. 2017;377:13-27. doi: 10.1056/NEJMoa1614362

4. Corazziari E. Definition and epidemiology of functional gastrointestinal disorders. Best Pract Res Clin Gastroenterol. 2004;18:613-631 doi: 10.1016/j.bpg.2004.04.012.

5. Nurko S, Di Lorenzo C. Functional abdominal pain: time to get together and move forward. J Pediatr Gastroenterol Nutr. 2008;47:679-680. doi: 10.1097 / MPG.0b013e31818936d1

6. Koppen IJ, Nurko S, Saps M, Di Lorenzo C, Benninga MA. The pediatric Rome IV criteria: what's new? Expert Rev Gastroenterol Hepatol. 2017 Mar;11(3):193-201. doi: 10.1080/17474124.2017.1282820. Epub 2017 Jan 24.

7. Chumpitazi BP, Shulman RJ. Underlying molecular and cellular mechanisms in childhood irritable bowel syndrome. Mol Cell Pediatr. 2016;3(1):1-9. doi: 10.1186/ s40348-016-0036-8.

8. Giannetti E, de'Angelis G, Turco R, Campanozzi A, Pensabene L, Salvatore S et al. Subtypes of irritable bowel syndrome in children: prevalence at diagnosis and at followup. J Pediatr. 2014;164(5):1099-1103. doi: 10.1016/j. jpeds.2013.12.043.

9. Belmer SV, Khavkin AI, Pechkurov DV. [Functional constipation in children (Look through the Rome consensus IV)]. Lechaschi Vrach. 2017;2:54-58.[Article in Russian].

10. Teitelbaum JE, Sinha P, Micale M, Yeung S, Jaeger J. Obesity is related to multiple functional abdominal diseases. J Pediatr. 2009;154(3):444-446. doi: 10.1016/j. jpeds.2008.09.053.

11. Galai T, Moran-Lev H, Cohen S, Ben-Tov A, Levy D, Weintraub $\mathrm{Y}$ et al. Higher prevalence of obesity among children with functional abdominal pain disorders. BMC Pediatr. 2020;20(1):1-6. doi: 10.1186/s12887-020-02106-9.

12. Pawłowska K, Umławska W, Iwańczak B. A link between nutritional and growth states in pediatric patients with functional gastrointestinal disorders. J Pediatr. 2018;199:171177. doi: 10.1016/j.jpeds.2018.02.069.

13. Tambucci R, Quitadamo P, Ambrosi M, De Angelis P, Angelino G, Stagi S. et al. Association between obesity/ overweight and functional gastrointestinal disorders in children. J Pediatr Gastroenterol Nutr. 2019;68(4):517-520. doi: 10.1097/MPG.0000000000002208.

14. Phatak UP, Pashankar DS. Prevalence of functional gastrointestinal disorders in obese and overweight children. Int J Obes (Lond). 2014;38(10):1324-1327. doi: 10.1038/ ijo.2014.67.

15. Fishman L, Lenders C, Fortunato C, Noonan C, Nurko $\mathrm{S}$. Increased prevalence of constipation and fecal soiling in a population of obese children. J Pediatr. 2004;145:253-254. doi: 10.1016/j.jpeds.2004.04.022

16. Pashankar DS, Loening-Baucke V. Increased prevalence of obesity in children with functional constipation evaluated in an academic medical center. Pediatrics. 2005;116(3):e377-e380. doi: 10.1542/peds.2005-0490.

17. Misra S, Lee A, Gensel K. Chronic constipation in overweight children. J Parenter Enteral Nutr. 2006;30(2):8184. doi: 10.1177/014860710603000281.

18. vd Baan-Slootweg OH, Liem O, Bekkali $\mathrm{N}$, van Aalderen WMC, Pels TH et al. Constipation and colonic transit times in children with morbid obesity. J Pediatr Gastroenterol Nutr. 2011;52(4):442-445. doi: 10.1097/ MPG.0b013e3181ef8e3c.

19. Rybochkina AV, Dmitrieva TG, Innokentieva NN. [Pathology of digestive organs in children with obesity in Sakha Republic (Yakutia)]. Vestnik of North-Eastern Federal University. Medical sciences. 2017;4(9):85-88. [Article in Russian].

20. Çağan Appak Y, Karakoyun M, Koru T, Baran M. Dietary properties and anthropometric findings of children with functional constipation: a cross-sectional study. Arch Argent Pediatr. 2019;117(3):e224-e231. doi: 10.5546/aap.2019.eng. e224.

21. Kelishadi R, Ardalan G, Gheiratmand R, Majdzadeh R, Hosseini M, Gouya MM et al. Thinness, overweight and obesity in a national sample of Iranian children and adolescents: CASPIAN Study. Child Care Health Dev. 2008;34(1):44-54. doi: 10.1111/j.1365-2214.2007.00744.x.

22. CostaML, Oliveira JN, Tahan S, Morais MB. Overweightand constipation in adolescents. BMC Gastroenterol. 2011;17;11:40. doi: 10.1186/1471-230X-11-40.

23. Koppen IJ, Velasco-Benítez CA, Benninga MA, Di Lorenzo C, Saps M. Is there an association between functional constipation and excessive bodyweight in children? J Pediatr. 2016;171:178-182.e1. doi: 10.1016/j.jpeds.2015.12.033.

24. Macêdo MIP, Albuquerque MDFM, Tahan S, de Morais MB. Is there any association between overweight, physical activity, fat and fiber intake with functional constipation in adolescents? Scand J Gastroenterol. 2020;55(4):414-420. doi: 10.1080/00365521.2020.1749878.

25. Kiefte-de Jong JC, de Vries JH, Escher JC, Jaddoe VWV, Hofman A, Raat $\mathrm{H}$ et al. Role of dietary patterns, sedentary behaviour and overweight on the longitudinal development of childhood constipation: the Generation R study. Matern Child Nutr. 2013;9(4):511-23. doi: 10.1111/j.17408709.2011.00395.x.

26. Chien LY, Liou YM, Chang P. Low defaecation frequency in Taiwanese adolescents: association with dietary intake, physical activity and sedentary behaviour. J Paediatr Child Health. 2011;47(6):381-6. doi: 10.1111/j.14401754.2010.01990.x.

27. Ho W, Spiegel BM. The relationship between obesity and functional gastrointestinal disorders: causation, association, or neither? Gastroenterol Hepatol (N Y). 2008 Aug;4(8):572-8.

28. Gurova MM, Novikova VP, Khavkin AI. The state of gut microbiota and clinical-metabolic features in children with overweight and obesity. Russian journal of evidence-based gastroenterology. 2018;7(3):4-10

29. Bonilla S, Wang D, Saps M. Obesity predicts persistence of pain in children with functional gastrointestinal disorders. Int J Obes (Lond). 2011;35:517-21. doi: 10.1038/ijo.2010.245. 30. Koppen IJ, Kuizenga-Wessel S, Saps M, Di Lorenzo $\mathrm{C}$, Benninga MA, van Etten-Jamaludin FS et al. Functional defecation disorders and excessive body weight: a systematic review. Pediatrics. 2016;138:pii:e20161417. doi: 10.1542/ peds.2016-1417.

31. Malaty H, Abudayyeh S, Fraley K, Graham DY, Gilger MA, Hollier DR. Recurrent abdominal pain in school children: effect of obesity and diet. Acta Paediatr. 2007;96:572-576. 
doi: 10.1111/j.1651-2227.2007.00230.x.

32. Pickett-Blakely O. Obesity and irritable bowel syndrome: a comprehensive review. Gastroenterol Hepatol (N Y). 2014;10(7):411-416.

33. Pavlovskaya EV, Strokova TV, Surkov AG. Et al. Dietary characteristics in children with overweight and obesity. Vopr Pitan. 2015;84(S5):58.

34. Schnabel L, Buscail C, Sabate, Bouchoucha JMM, KesseGuyotE, Allès B. et al. Association between ultra-processed food consumption and functional gastrointestinal disorders: results from the French NutriNet-Santé cohort. Am J Gastroenterol . 2018;113(8):1217-1228. doi: 10.1038/s41395-018-0137-1.

35. Barrett JS, Gibson PR. Fermentable oligosaccharides, disaccharides, monosaccharides and polyols (FODMAPs) and nonallergic food intolerance: FODMAPs or food chemicals? Ther Adv Gastroenterol. 2012;5:261-268. doi: $10.1177 / 1756283$ X11436241.

36. Ozaki RKF, Speridião PDGL, Soares ACF, de Morais MB. Intestinal fructose malabsorption is associated with increased lactulose fermentation in the intestinal lumen. J Pediatr (Rio J). 2018;94(6):609-615. doi: 10.1016/j.jped.2017.08.006.

37. Fodor I, Man SC, Dumitrascu DL. Low fermentable oligosaccharides, disaccharides, monosaccharides, and polyols diet in children. World J Clin Cases. 2019;7(18):2666. doi: 10.12998/wjcc.v7.i18.2666.

38. Nalyotov AV, Vunichenko YS. The using of lowFODMAP diet is an important step in the treatment of children with irritable bowel syndrome. Pediatrician (St. Petersburg). 2017;8(6):94-98. doi: 10.17816/PED8694-98

39. Feinle-Bisset C, Azpiroz F. Dietary lipids and functional gastrointestinal disorders. Am. J. Gastroenterol. 2013;108:737747. doi: 10.1038/ajg.2013.76.

40. Accarino AM, Azpiroz F, Malagelada JR. Modification of small bowel mechanosensitivity by intestinal fat. Gut. 2001;48:690-695. doi: 10.1136/gut.48.5.690.

41. Levy RL, Linde JA, Feld KA, Crowell MD, Jeffery RW et al. The association of gastrointestinal symptoms with weight, diet, and exercise in weight-loss program participants. Clin Gastroenterol Hepatol. 2005;3(10):992-996. doi: 10.1016/ s1542-3565(05)00696-8.

42. Piccoli de Mello P, Eifer DA, Daniel de Mello E. Use of fibers in childhood constipation treatment: Systematic review with meta-analysis. J. Pediatr. 2018;94:460-470. doi: 10.1016/j.jped.2017.10.014.

43. Khavkin AI, Fayzullina RA, Belmer SV, Gorelov AV, Zakharova IN, Zvyagin AA et al. Diagnosis and tactics of treatment of children with functional constipation (recommendations of the Society of Paediatric Gastroenterologists). Pediatric Nutrition. 2014;12(4):49-63.

44. He J, Cai Z, Fan, X. Prevalence of binge and loss of control eating among children and adolescents with overweight and obesity: An exploratory meta-analysis. Int J Eat Disord. 2017;50(2):91-103. doi:10.1002/eat.22661.

45. Decaluwe V, Braet C, Fairburn CG. Binge eating in obese children and adolescents. Int J Eat Disord 2003;33:78-84. doi: 10.1038/sj.ijo.0802233.

46. Moayyedi P. The epidemiology of obesity and gastrointestinal and other diseases: an overview. Dig Dis Sci. 2008;53:2293-2299. doi: 10.1007/s10620-008-0410-z. 47. Rankin J, Matthews L, Cobley S, Han A, Sanders R, Wiltshire HD et al. Psychological consequences of childhood obesity: psychiatric comorbidity and prevention. Adolesc Health Med Ther. 2016;7:125-146. doi: 10.2147/AHMT.S101631.
48. Larina NG. Food behaviour, lifestyle, and psychological status of adolescents with obesity and autonomic dysfunction. Vestnik NovSU. 2017;8(106):58-61.

49. Yacob D, Di Lorenzo C, Bridge JA, Rosenstein PF, Onorato M, Bravender $\mathrm{T}$ et al. Prevalence of painpredominant functional gastrointestinal disorders and somatic symptoms in patients with anxiety or depressive disorders. J Pediatr 2016;163:767-770. doi: 10.1016/j.jpeds.2013.02.033. 50. Wouters MM, Boeckxstaens GE. Is there a causal link between psychological disorders and functional gastrointestinal disorders? Expert Rev Gastroenterol Hepatol 2016;10:5-8. doi: 10.1586/17474124.2016.1109446.

51. Vu J, Kushnir V, Cassell B, Gyawali CP, Sayuk GS. The impact of psychiatric and extraintestinal comorbidity on quality of life and bowel symptom burden in functional gastrointestinal disorders. Neurogastroenterol Motil 2014;26:1323-1332. doi: 10.1111/nmo.12396.

52. Koloski NA, Jones M, Kalantar J, Weltman M, Zaguirre J, Talley NJ. The brain-gut pathway in functional gastrointestinal disorders is bidirectional: a 12-year prospective populationbased study. Gut. 2012;61(9):1284-1290. doi: 10.1136/ gutjnl-2011-300474.

53. Dinan TG, Quigley EM, Ahmed SM, Scully P, O’Brien S, O'Mahony $\mathrm{L}$ et al. Hypothalamic-pituitary-gut axis dysregulation in irritable bowel syndrome: plasma cytokines as a potential biomarker? Gastroenterology 2006;130:304311. doi: 10.1053/j.gastro.2005.11.033.

54. Iovino P, Tremolaterra F, Boccia G, Miele E, Ruju FM, Staiano A. Irritable bowel syndrome in childhood: visceral hypersensitivity and psychosocial aspects. Neurogastroenterol Motil. 2009;21(9):940-e974. doi: 10.1111/j.13652982.2009.01303.x.

55. Schneck AS, Anty R, Tran A, Hastier A, Amor IB, Gugenheim $J$ et al. Increased prevalence of irritable bowel syndrome in a cohort of French morbidly obese patients candidate for bariatric surgery. Obes Surg. 2016;26(7),15251530. doi: 10.1007/s11695-015-1907-0.

56. Delgado-Aros S, Camilleri M, Garcia MA, Burton D, Busciglio I. High body mass alters colonic sensory-motor function and transit in humans. Am J Physiol Gastrointest Liver Physiol. 2008;295:G382-8. doi: 10.1152/ajpgi.90286.2008.

57. Bouchoucha M, Fysekidis M, Rompteaux P, Airinei G, Sabate JM, Benamouzig R. Influence of age and body mass index on total and segmental colonic transit times in constipated subjects. J Neurogastroenterol Motil. 2019;25(2):258-266. doi: $10.5056 /$ jnm 18167.

58. Gomez-Pinilla PJ, Gibbons SJ, Sarr MG, Kendrick ML, Shen KR, Cima RR et al. Changes in interstitial cells of Cajal with age in the human stomach and colon. Neurogastroenterol Motil. 2011;23(1):36-44. doi: 10.1111/j.1365-2982.2010.01590.x.

59. Talley NJ, Howell S, Poulton R. Obesity and chronic gastrointestinal tract symptoms in young adults: a birth cohort study. Am J Gastroenterol. 2004;99(9):1807-1814. doi: 10.1111/j.1572-0241.2004.30388.x.

60. Drapkina OM, Korneeva ON. [Gut microbiota and obesity: Pathogenetic relationships and ways to normalize the intestinal microflora]. Ter Arkh. 2016;88(9):135-142. doi: 10.17116/terarkh2016889135-142. [Article in Russian].

61. Da Silva CC, Monteil MA, Davis EM. Overweight and obesity in children are associated with an abundance of Firmicutes and reduction of Bifidobacterium in their gastrointestinal microbiota. Child Obes. 2020;16(3):204-210. doi: 10.1089/chi.2019.0280. 
62. Belkova NL, Nemchenko UM, Pogodina AV, Feranchuk SI, Romanitsa AI, Novikova EA et al. Composition and structure of gut microbiome in adolescents with obesity and different breastfeeding duration. Bull Exp Biol Med. 2019;167(6):759-762. doi: 10.1007/s10517-019-04617-7.

63. Rajilic-Stojanovic M, Biagi E, Heilig HG, Kekkonen RA, Tims S, de Vos WM. Global and deep molecular analysis of microbiota signatures in fecal samples from patients with irritable bowel syndrome. Gastroenterology. 2011;141:17921801. doi:10.1053/j.gastro.2011.07.043.

64. Saulnier DM, Riehle K, Mistretta TA, Diaz MA, Mandal $\mathrm{D}$, Raza $\mathrm{S}$ et al. Gastrointestinal microbiome signatures of pediatric patients with irritable bowel syndrome. J Gastroenterology. 2011;141(5):1782-1791. doi: 10.1053/j. gastro.2011.06.072.

65. Horvath A, Dziechciarz P, Szajewska H. Meta-analysis: Lactobacillus rhamnosus GG for abdominal pain-related functional gastrointestinal disorders in childhood. Aliment Pharmacol Ther. 2011;33(12):1302-1310. doi: 10.1111/j.13652036.2011.04665.x.

66. Guandalini S, Magazzu G, Chiaro A, La Balestra V, Di Nardo G, Gopalan S et al. VSL\#3 improves symptoms in children with irritable bowel syndrome: a multicenter, randomized, placebo-controlled, double-blind, crossover study. J Pediatr Gastroenterol Nutr 2010;51:24-30. doi: 10.1097/MPG.0b013e3181ca4d95.

67. Giannetti E, Maglione M, Alessandrella A, Strisciuglio C, De Giovanni D, Campanozzi A et al. A mixture of 3 Bifidobacteria decreases abdominal pain and improves the quality of life in children with irritable bowel syndrome: a multicenter, randomized, double-blind, placebo-controlled, crossover trial. J Clin Gastroenterol 2017;51:e5-e10. doi: 10.1097/MCG.0000000000000528.

68. de Meij TGJ, de Groot EFJ, Eck A, Budding AE, Kneepkens $\mathrm{CM}$, Benninga $\mathrm{MA}$ et al. Characterization of microbiota in children with chronic functional constipation. PLoS ONE. 2016;11(10):e0164731. doi: 10.1371/journal.pone.0164731.

69. Zhu L, Liu W, Alkhouri R, Baker RD, Bard JE, Quigley EM et al. Structural changes in the gut microbiome of constipated patients. Physiol Genomics. 2014;46:679-686. doi: 10.1152/physiolgenomics.00082.2014.

70. Zoppi G, Cinquetti M, Luciano A, Benini A, Muner A, Bertazzoni Minelli E.. The intestinal ecosystem in chronic functional constipation. Acta Paediatr. 1998;87:836-841. doi: 10.1080/080352598750013590.

71. Wojtyniak K., Szajewska H. Systematic review: probiotics for functional constipation in children. Eur $\mathrm{J}$ Pediatr. 2017;176(9):1155-1162. doi: 10.1007/s00431-017-2972-2. 